\title{
Application and Impact of Run-In Studies for the Evaluation of Statin Efficacy and Safety
}

\author{
Michael Fralick, MD, MSc ${ }^{1,2,3}$, Jerry Avorn, $M D^{1,3}$, Jessica M. Franklin, $P h D^{1,3}$, Emily Bartsch, $B M S c^{3}$, \\ Abdurrahman Abdurrob, BS ${ }^{3}$, and Aaron S. Kesselheim, MD, JD, MPH ${ }^{1,3}$

\begin{abstract}
'Program on Regulation, Therapeutics, and Law (PORTAL), Division of Pharmacoepidemiology and Pharmacoeconomics, Department of Medicine, Brigham and Women's Hospital and Harvard Medical School, Boston, MA, USA; 'Eliot Phillipson Clinician-Scientist Training Program, Toronto, Canada; ${ }^{3}$ Division of Pharmacoepidemiology and Pharmacoeconomics, Department of Medicine, Brigham and Women's Hospital and Harvard Medical School, Boston, MA, USA.
\end{abstract}

KEY WORDS: run-in; statin; clinical trial; study design.

J Gen Intern Med 33(6):792-4

DOI: $10.1007 / \mathrm{s} 11606-018-4349-2$

(c) Society of General Internal Medicine 2018

$\mathrm{S}$ ome randomized controlled trials (RCTs) include a prerandomization run-in phase. ${ }^{1}$ During this phase, eligible patients are typically administered a drug or placebo to exclude non-adherent patients, placebo responders, or patients who do not respond to or tolerate the active drug prior to trial initiation. ${ }^{1,2}$ Our review of DPP4 inhibitors found that trials with run-in phases provided findings of efficacy and safety similar to those seen in trials of the same medications without run-in phases. ${ }^{3}$ We sought to determine if this observation also applied to statin medications.

\section{METHODS}

\section{Data Source}

To systematically identify relevant clinical trials of statins, we reviewed all trials listed in a recently published comprehensive meta-analysis of statins for the prevention of cardiovascular disease in adults. ${ }^{4}$ That study reviewed trials through June 2016.

\section{Study Endpoints}

We compared all-cause mortality among RCTs designed with and without run-in phases as well as key adverse events. Elevated aminotransferase levels were selected as the adverse event of interest since this was widely reported, is a common adverse event among statins, and may herald important patient morbidity.

\section{Data Extraction}

Data were extracted from each article independently by two investigators (EB, MF) with differences resolved by consensus.

Published online March 8, 2018

\section{Statistical Analysis}

Efficacy results (i.e., overall mortality) and safety results (i.e., elevated aminotransferases) from each trial for each drug were pooled separately using a random-effects model. ${ }^{5}$ To determine the impact of the run-in phase, meta-regression was performed separately for the efficacy endpoint and the safety endpoint using Stata (IC version 14.2, College Station, TX).

Role of the Funding Source. The funding source had no role in the study design; the collection, analysis, and interpretation of data; the writing of the report; or the decision to submit the paper for publication.

\section{RESULTS}

We identified 16 RCTs of statins, 10 with and 6 without run-in phases. Characteristics of patients enrolled and randomized in trials with or without run-in phases were similar (Table 1). Demographic characteristics of patients who entered the runin phase, but were excluded and not randomized, were not reported in the manuscripts of the articles we identified. Trials with run-in phases screened more patients for inclusion and were generally larger than trials without run-in phases (Table 1). For trials with run-in phases, the mean duration of the run-in phases was 6.2 weeks (standard deviation $[\mathrm{SD}]=3.1$ ). Active medications were administered only rarely during the run-in phases ( $10 \%$ of studies). Half of all trials with run-in phases did not indicate why patients were excluded after the run-in phases, but when it was reported, poor adherence was the most common reason (Table 1).

The reduction in overall mortality was similar for trials with a run-in phase compared to trials without a run-in phase (risk ratio [RR] $0.87,95 \%$ CI $0.80-0.95, I^{2}=5 \%$ versus $\mathrm{RR}=0.76$, 95\% CI 0.57-1.01, $I^{2}=0 \%$, respectively; $p=0.51$ for the difference in RRs) (Fig. 1). The rate of elevated aminotransferase levels was also similar for patients in trials with run-in phases compared to trials without run-in phases (RR 1.10, 95\% CI $0.89-1.37, I^{2}=0 \%$ versus RR $1.18,95 \%$ CI $0.65-$ $2.16, I^{2}=0 \%$, respectively; $p=0.76$ for the difference in RRs). 
Table 1 Trial and Patient-Level Characteristics

\begin{tabular}{|c|c|c|c|}
\hline & Run-in $(N=10)$ & No run-in $(N=6)$ & $p$ \\
\hline Age in years, mean (SD) & $60.4(3.7)$ & $57.8(3.1)$ & 0.164 \\
\hline Percent male sex, mean (SD) & $69(22)$ & $58(34)$ & 0.411 \\
\hline Percent Caucasian race, mean (SD) & $68(37)$ & $88(13)$ & 0.272 \\
\hline Baseline BMI, mean (SD) & $27.0(1.6)$ & $28.5(1.9)$ & 0.125 \\
\hline Percent smoker, mean (SD) & $21(12)$ & $24(11)$ & 0.669 \\
\hline Systolic blood pressure mmHg, mean (SD) & $137(11)$ & $136(7)$ & 0.945 \\
\hline Patients assessed for trial, mean (SD) & $35,053(39,525)$ & 3331 (3919) & 0.144 \\
\hline Patients randomized, mean (SD) & $6699(5756)$ & $903(976)$ & 0.03 \\
\hline Percent of patients completing trial, mean (SD) & $88(13)$ & $78(15)$ & 0.239 \\
\hline Percent of trials that were blinded & 90 & 100 & 1 \\
\hline Percent of trials with concealed allocation & 100 & 100 & - \\
\hline Duration of follow-up in weeks, mean (SD) & $215(70)$ & $181(44)$ & 0.356 \\
\hline Run-in duration in weeks, mean (SD) & $6.2(3.1)$ & NA & \\
\hline \multicolumn{4}{|l|}{ Intervention administered during run-in phase } \\
\hline Placebo & $40 \%$ & NA & \\
\hline Statin and blood pressure-lowering medication & $10 \%$ & NA & \\
\hline Diet & $30 \%$ & NA & \\
\hline Placebo and diet & $10 \%$ & NA & \\
\hline Unclear & $10 \%$ & NA & \\
\hline \multicolumn{4}{|l|}{ Reason for exclusion after run-in } \\
\hline Not reported & $50 \%$ & NA & \\
\hline Poor adherence & $37 \%$ & NA & \\
\hline Poor adherence and/or not well tolerated & $13 \%$ & NA & \\
\hline
\end{tabular}

SD standard deviation, BMI body mass index $\left(\mathrm{kg} / \mathrm{m}^{2}\right)$, NA not applicable

Relative Risk $(95 \% \mathrm{Cl})$

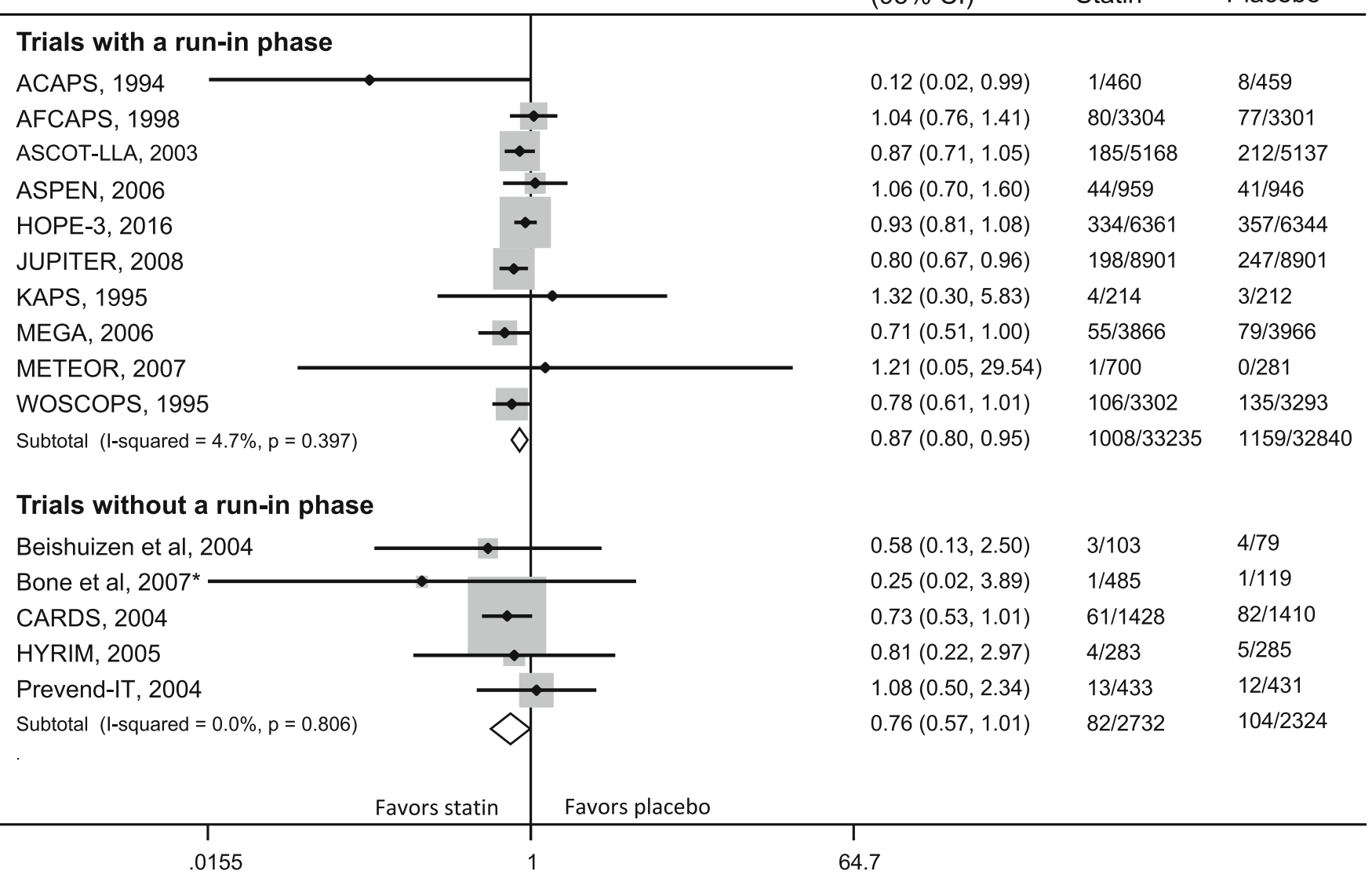

Legend - Events are number of patients with an event. $\mathrm{N}$ - study sample size. $95 \% \mathrm{Cl}-95 \%$ confidence interval. $* 1$ event was imputed for both the statin arm and placebo arm since both study arms had zero events. Weights are from random effects analysis

Fig. 1 Meta-analysis: all-cause mortality in randomized trials of statins with and without run-in phases. 
Although not statistically significant, trials with a run-in phase had higher rates of trial completion (Table 1).

\section{DISCUSSION}

As we found in our review of DPP4 inhibitors, trials of statins with run-in phases provided results that were nearly identical in key efficacy and safety outcomes to those in trials of statins without run-in phases.

These findings provide additional evidence that run-in phases might not be a good use of scarce clinical trial resources. However, we were limited by the number of available clinical trials of statins, and thus our study may have been underpowered. For example, trials of statins with run-in phases appeared to have a higher percentage of patients completing the trial compared to trials of statins without run-in phases (i.e., $88 \%$ versus $78 \%$, respectively), but this did not reach statistical significance. We did not observe this difference in our study of DPP4 inhibitors, but those studies were shorter (about 30 weeks) in duration than the trials of statins (about 200 weeks). Thus, run-in phases might improve rates of completion in trials of longer duration. Further research will be required to determine if this observation is replicable.

Corresponding Author: Michael Fralick, MD, MSc; Division of Pharmacoepidemiology and Pharmacoeconomics, Department of MedicineBrigham and Women's Hospital and Harvard Medical School, Boston, MA, USA (e-mail: mif823@mail.harvard.edu).
Authors' Contributions Study concept and design: Fralick $M$, Kesselheim A, and Avorn $J$

Acquisition of data: Fralick M, Abdurrob A, and Bartsch $E$

Analysis/interpretation of data: Fralick M, Kesselheim A, Franklin J, Avorn J, and Bartsch E

Drafting of the manuscript: Fralick $M$

Critical revision of the manuscript: Kesselheim A, Franklin J, Avorn J, Abdurrob A, and Bartsch E

Statistical analysis: Fralick $M$ and Franklin $J$

Funding Dr. Fralick receives funding from the Eliot Phillipson Clinician-Scientist Training Program and the Clinician Investigator Program at the University of Toronto and from The Detweiler Travelling Fellowship funded by the Royal College of Physicians and Surgeons of Canada. This study is also funded by The Laura and John Arnold Foundation. Dr. Kesselheim's work is also supported by the Harvard Program in Therapeutic Science and the Engelberg Foundation.

\section{Compliance with Ethical Standards:}

Conflict of Interest: Dr. Franklin is the principal investigator on a grant from Merck. All remaining authors declare that they do not have a conflict of interest.

\section{REFERENCES}

1. Pablos-Méndez A, Barr RG, Shea S. Run-in periods in randomized trials: implications for the application of results in clinical practice. JAMA. 1998;279(3):222-225.

2. Rothwell PM. Factors that can affect the external validity of randomised controlled trials. PLoS Clin Trials. 2006;1(1):e9.

3. Fralick M, Avorn J, Franklin JM, Abdurrahman A, Kesselheim AS. Application and impact of run-in studies. JGIM. 2018.

4. Chou R, Dana T, Blazina I, Daeges M, Jeanne TL. Statins for prevention of cardiovascular disease in adults. JAMA. 2016;316(19):2008-2024.

5. Dersimonian R, Laird N. Meta-analysis in clinical trials. Stat Med. 1986; 188: 177-188. 